\title{
ANÁLISE DA INSERÇÃO DA SAÚDE NOS ESTUDOS DE IMPACTO AMBIENTAL DOS LICENCIAMENTOS DAS BARRAGENS DE REJEITO DA SAMARCO, COMPLEXO DE GERMANO, MARIANA, MINAS GERAIS
}

\author{
ANALYSIS OF THE INCLUSION OF HEALTH MATTERS IN THE ENVIRONMENTAL \\ IMPACT STUDIES CARRIED OUT FOR LICENSES TO THE TAILINGS DAMS OPERATED \\ BY SAMARCO, IN GERMANO COMPLEX, MARIANA, MINAS GERAIS
}

\author{
Mariana dos Santos Lima Luz \\ Geógrafa e Bacharel em Gestão Ambiental \\ marianaluz@hotmail.com
}

Flavio Fernando Batista Moutinho

UFF e Fundação Municipal de Saúde de Niterói flaviomoutinho@id.uff.br

\begin{abstract}
RESUMO
A mineração é uma atividade que necessita de licença ambiental para funcionar. Apesar da legislação vigente prever que aspectos de saúde sejam contemplados nos Estudos de Impacto Ambiental (EIA) no âmbito do licenciamento ambiental, isso ainda é muito incipiente. O presente artigo teve como objetivo analisar como os aspectos relacionados à saúde humana foram contemplados nos EIA referentes aos licenciamentos das barragens de rejeitos de mineração da empresa Samarco, Complexo de Germano, município de Mariana, estado de Minas Gerais, que passou por um grave desastre ambiental em 2015. Para tanto, foram analisados o EIA elaborado visando o licenciamento da barragem de rejeitos de minério de ferro de Fundão, rompida em 2015, e o EIA para instalação do sistema de contenção de rejeitos Alegria Sul, que veio substituir o anterior. Trata-se de uma pesquisa qualitativa de análise documental onde foi utilizada uma matriz de análise desenvolvida especificamente para esse fim. O estudo comparativo entre os dois EIA permitiu concluir que a inserção das questões de saúde nos dois estudos ofereceu resultados sobre a inserção da saúde similares e incipientes, com leve superioridade do EIA de Alegria Sul. A pesquisa ajudou a compreender as fragilidades que existem na participação do setor de saúde no licenciamento ambiental de barragens de rejeitos de mineração.
\end{abstract}

Palavras-chave: Licenciamento ambiental. Saúde ambiental. Mineração.

\begin{abstract}
Mining is an activity requiring an environmental license in order to be carried out. Although the law in force provides for the inclusion of health concerns in the Environmental Impact Studies (EIS) in the scope of environmental licenses, this practice is still very insipient. This article aims at analyzing how human health related aspects were contemplated in the EIS regarding the licenses issued for the mining tailings dams operated by the company Samarco, in Germano Complex, located in the city of Mariana, state of Minas Gerais, which suffered a serious environmental disaster in 2015. Therefore, we analyzed the EIS prepared for the licensing of the iron ore tailings dam of Fundão, collapsed in 2015, and the EIS for the installation of the Alegria Sul's tailings containment system, which replaced the previous one. This is a documental analysis qualitative research, where an analysis matrix specifically developed for that purpose was used. The comparative study between both EIS allowed us to conclude that the inclusion of health matters in both studies provided similar and incipient results as regards said inclusion, the Alegria Sul's EIS being slightly superior. This research helped us understand the weaknesses existing in the health sector stake for environmental licenses granted to mining tailings dams.
\end{abstract}

Keywords: Environmental licenses. Environmental health. Mining. 


\section{INTRODUÇÃO}

A avaliação de impactos e o licenciamento ambiental de atividades efetiva ou potencialmente poluidoras são instrumentos da Política Nacional de Meio Ambiente (BRASIL, 1981). No caso da mineração, acredita-se que os estudos de impacto ambiental devam ser capazes de fornecer um prognóstico sobre os impactos negativos das barragens de rejeito em relação ao ambiente e à saúde potencialmente afetada pelo empreendimento minerador mas, como destacado por Mansur et al. (2016), os estudos e avaliações de impacto dos processos de licenciamento ambiental têm sido ineficazes.

Ainda que a definição de impacto ambiental da Resolução Conama $n^{\circ}$ 001/1986 faça referência aos aspectos de saúde e que a Resolução Conama $n^{\circ} 237 / 97$ estabeleça direitos ao órgão ambiental de suspender ou cancelar uma licença em caso de ocorrência de graves riscos ambientais e de saúde, na prática, os estudos e relatórios ainda apresentam o tema de forma simplificada, pouco aprofundada, com carência de informações relevantes. Em geral, os aspectos de saúde não são abordados em primeiro plano, mas como consequência indireta dos impactos ambientais (BARBOSA et al., 2010).

Não existe uma ferramenta de avaliação de impacto na saúde específica para os processos de licenciamento ambiental, sendo as questões relativas à saúde humana consideradas como um subcomponente no capítulo de Diagnóstico do Meio Socioeconômico. (MINISTÉRIO DA SAÚDE, 2014). Quando solicitado pelo órgão ambiental responsável pelo licenciamento ambiental, o setor saúde insere suas recomendações dentro de um formato já padronizado que busca propor medidas preventivas, de promoção e proteção à saúde, e consequentemente a redução dos impactos na saúde humana (SILVEIRA; ARAÚJO NETO, 2013).

Rompimento de barragem de mineração geralmente ocorrem com a ruptura da estrutura ocasionados por fatores como manutenção inadequada do sistema de drenagem, uso de metodologias obsoletas no processo construtivo, falhas de monitoramento, sobrecarga, dentre outros (RICO et al, 2008). A dinâmica econômica do setor é outro fator que pode influenciar na ocorrência de tais desastres (MANSUR et al., 2016), já que a demanda da indústria siderúrgica e a alta internacional nos preços dos minerais leva à intensificação da produção (MILANEZ et al., 2016). De acordo com Porto (2016) a ocorrência de rompimentos é comum nessas ocasiões de aumento da produção já que há o consequente aumento da quantidade de rejeitos gerados.

O método obsoleto de construção de barragens de rejeitos pelo alteamento a montante, de mais barata instalação, está relacionado à maioria dos casos de ruptura dessas estruturas. Práticas mais sustentáveis, como a empilhagem a seco ou a separação eletromagnética, poderiam restringir ou até mesmo eliminar a existência das barragens, entretanto, o que se observa é a viabilização de enormes quantidades de áreas mineradas que ampliam a quantidade de rejeitos gerados e utilizam essas técnicas obsoletas para construção de barragens de contenção de rejeitos em detrimento alternativas mais seguras (PORTO, 2016).

Os desastres com rompimento de barragens de rejeitos de mineração são comuns no Brasil, especialmente em Minas Gerais, onde o primeiro registro data de 1986, episódio que vitimou fatalmente sete pessoas (LACAZ et al., 2017). Além desse, houve em Nova Lima, em 2001, em Miraí, em 2007, em Congonhas, em 2008 e em Itabirito, em 2014 (LACAZ et al., 2017). Posteriormente, em 2019, foi registrado mais um grave desastre, no município de Brumadinho, também em Minas Gerais, que vitimou fatalmente mais de 200 pessoas, deixando dezenas de desaparecidos (GMG, 2019).

O desastre ocorrido em Mariana, em 2015, com o rompimento da barragem de rejeitos da mineradora Samarco, denominada Fundão, no Complexo de Germano, é considerado o maior desastre socioambiental envolvendo o setor de mineração, tendo lançado no ambiente cerca de 45 milhões de metros cúbicos de rejeito (IBAMA, 2016), afetando o rio Doce e diversas localidades dos estados de Minas Gerais e Espírito Santo (BORGES, 2018). Cabe destacar que o rejeito possui partículas em suspensão e dissolvidas e pode ser altamente tóxico, com presença de reagentes e metais pesados (LOZANO, 2006)

Com o desastre, para que a atividade voltasse a funcionar, havia a necessidade de um novo local para dispor os rejeitos. Assim, a empresa optou pela utilização da barragem já existente denominada Alegria Sul (ARCADIS, 2016).

Pode-se considerar que a possibilidade de ocorrência de desastres é motivo de preocupação no que 
tange à Saúde Pública já que eles podem ter potencial de causar graves impactos de curto, médio e longo prazos à saúde das populações atingidas (FREITAS; AMORIM, 2001). O potencial de repercussão sobre o setor saúde dos desastres envolvendo a mineração, por exemplo, é muito grande. Fatores como o atendimento às necessidades imediatas das vítimas pós desastre, elaboração de programas de gestão de riscos e realização de melhorias estruturais na rede de serviços de saúde danificadas ou destruídas devem ser considerados (FREITAS, 2018).

Assim sendo, o presente artigo objetivou analisar como os aspectos relacionados à saúde humana foram contemplados nos Estudos de Impacto Ambiental referentes aos licenciamentos das barragens de rejeitos de mineração da empresa Samarco, Complexo de Germano, Mariana, Minas Gerais. Para tanto, foram analisados o Estudo de Impacto Ambiental (EIA) elaborado objetivando à obtenção da Licença Ambiental Prévia da barragem de rejeitos de minério de ferro de Fundão e o EIA apresentado pela empresa visando o licenciamento ambiental do sistema de disposição de rejeitos Alegria Sul.

\section{METODOLOGIA}

Para o alcance dos objetivos almejados optou-se por uma pesquisa com metodologia qualitativa tendo como base a análise documental. Qualitativa por buscar descrever e compreender um fenômeno que não pode ser quantificado, que envolve valores, atitudes, motivos e significados (MINAYO, 2001). A estratégia da análise documental tem nos documentos o objeto de investigação, documentes estes de onde se pode obter as informações para esclarecer os fenômenos em estudo (FIGUEIREDO,2007).

Nesse contexto, os documentos que serviram de base para a análise foram os estudos de impacto ambiental desenvolvidos para o licenciamento prévio da Barragem de Rejeito de Germano, de 2005, e para o Sistema de Disposição de Rejeito Alegria Sul, de 2016 (SIAM,2019). A partir dos documentos elencados foi realizada a chamada leitura flutuante (BARDIN, 1977), visando estabelecer um primeiro contato com os documentos citados para conhecer seus conteúdos e selecionar os de interesse da pesquisa. Foram selecionados, então, os capítulos de Descrição do Projeto, de Diagnóstico do Meio Socioeconômico e de Avaliação de Impactos Ambientais.

No capítulo Descrição do Projeto foram consideradas a presença de profissionais da área da saúde na equipe de desenvolvimento do EIA, a existência de inventário de substâncias químicas e os riscos advindos dessas substâncias e, por fim, as estimativas de aplicação de recursos na área da saúde.

No capítulo Diagnóstico Socioeconômico foi analisada a existência de dados demográficos da população da área de influência do projeto, tais como, densidade populacional, tamanho, taxa de natalidade, divisão por sexo e idade, taxa de mortalidade e tendências demográficas. Analisou-se, também, a dinâmica de ocupação e uso do solo, os deslocamentos da população, a situação das habitações, transportes, emprego, acesso a serviços públicos, assistência social, existência e acesso a serviços de saúde, perfil epidemiológico e indicadores comportamentais (atividade física, tabagismo, dieta, uso de álcool, dentre outros).

Por fim, analisou-se a presença de dados sobre a qualidade da água, do ar e do solo, bem como sobre a participação popular. Além disso, buscou-se avaliar a percepção da população sobre os possíveis impactos à saúde e as medidas que envolvem a garantia de investimentos na saúde e a segurança, visando perceber a dimensão da geração de insegurança e expectativas.

No capítulo Avaliação de Impacto Ambiental considerou-se dados referentes a impactos e riscos à saúde da população, dos trabalhadores, a indicadores ambientais (qualidade de solo, água e ar), a indicadores de saúde (morbimortalidade, atendimentos ambulatoriais e hospitalares, dentre outros), e a indicadores socioeconômicos (escolaridade, emprego, renda, produto interno bruto, dentre outros).

Para avaliação foi desenvolvida uma matriz de análise (Quadro 1), adaptada de outras avaliações similares desenvolvidas no âmbito do licenciamento ambiental de atividades diversas da mineração, como os setores hidrelétrico e de petróleo (SORDIA; DIAZ, 1999; CANCIO, 2008; BARBOSA et al., 2012; MINISTÉRIO DA SAÚDE, 2014). 
Quadro 1 - Matriz de análise das questões de saúde nos estudos de impacto ambiental da Samarco para a barragem do Fundão (2005) e para o Sistema de Disposição de Rejeito - Alegria Sul (2016)

\begin{tabular}{|c|c|c|c|}
\hline \multirow[t]{2}{*}{ Categoria Operacional } & \multicolumn{3}{|c|}{ Explicitação do conteúdo no EIA } \\
\hline & Inexistente & $\begin{array}{l}\text { Parcialmente } \\
\text { Existente }\end{array}$ & Existente \\
\hline \multicolumn{4}{|c|}{ DESCRIÇÃO DO PROJETO } \\
\hline \multicolumn{4}{|c|}{\begin{tabular}{|l|l} 
Participação de profissionais com perfil específico da & \\
área de saúde &
\end{tabular}} \\
\hline \multicolumn{4}{|l|}{$\begin{array}{l}\text { Inventário das substâncias químicas relacionadas ao } \\
\text { empreendimento e os riscos à saúde associados }\end{array}$} \\
\hline \multicolumn{4}{|c|}{\begin{tabular}{|l|l|l} 
Estimativas de aplicação de recursos financeiros, & \\
direta ou indiretamente, na melhoria da saúde & \\
\end{tabular}} \\
\hline \multicolumn{4}{|c|}{ DIAGNÓSTICO DO MEIO SOCIOECONÔMICO } \\
\hline \multicolumn{4}{|c|}{$\begin{array}{l}\text { Características gerais da população (tamanho, } \\
\text { densidade, distribuição, idade e sexo e tendências } \\
\text { demográficas) }\end{array}$} \\
\hline \multicolumn{4}{|l|}{$\begin{array}{l}\text { Dinâmica de ocupação e uso do território, migrações e } \\
\text { deslocamentos populacionais }\end{array}$} \\
\hline \multicolumn{4}{|l|}{$\begin{array}{l}\text { Condições de habitação, situação de emprego, } \\
\text { infraestrutura de transportes, assistência social e } \\
\text { condições e acesso a serviços públicos }\end{array}$} \\
\hline \multicolumn{4}{|l|}{$\begin{array}{l}\text { Acesso a serviços e estabelecimentos de saúde e a } \\
\text { capacidade de suporte (infraestrutura, número de } \\
\text { leitos, profissionais de saúde, etc.) }\end{array}$} \\
\hline \multicolumn{4}{|l|}{$\begin{array}{l}\text { Perfil epidemiológico da população (difusão e } \\
\text { propagação de doenças, sua frequência, seu modo de } \\
\text { distribuição, sua evolução, etc.) }\end{array}$} \\
\hline \multicolumn{4}{|l|}{$\begin{array}{l}\text { Indicadores comportamentais, incluindo a dieta, o } \\
\text { tabagismo, a atividade física e o uso de álcool }\end{array}$} \\
\hline \multicolumn{4}{|l|}{$\begin{array}{l}\text { Condições ambientais como a qualidade do ar, água e } \\
\text { solo, a presença de áreas contaminadas }\end{array}$} \\
\hline \multicolumn{4}{|l|}{$\begin{array}{l}\text { Geração de insegurança - Percepção da população } \\
\text { sobre os efeitos na saúde decorrentes dos impactos } \\
\text { ambientais do empreendimento }\end{array}$} \\
\hline \multicolumn{4}{|l|}{$\begin{array}{l}\text { Geração de expectativa - Percepção da população } \\
\text { sobre as medidas de segurança e garantias de } \\
\text { investimentos na saúde }\end{array}$} \\
\hline \multicolumn{4}{|c|}{ AVALIAÇÃO DE IMPACTOS AMBIENTAIS } \\
\hline \multicolumn{4}{|c|}{\begin{tabular}{ll|l|l|} 
Identificação dos impactos à saúde dos trabalhadores & \\
e da população residente no entorno do & \\
empreendimento & & & \\
\end{tabular}} \\
\hline \multicolumn{4}{|l|}{$\begin{array}{l}\text { Impactos sobre indicadores de saúde (morbidade, } \\
\text { mortalidade, atendimentos ambulatorial e hospitalar, } \\
\text { etc.) }\end{array}$} \\
\hline \multicolumn{4}{|l|}{$\begin{array}{l}\text { Alteração na qualidade do solo, na qualidade da água } \\
\text { e do ar }\end{array}$} \\
\hline $\begin{array}{l}\text { Impactos sobre indicadores sociais e econômicos (PIB, } \\
\text { escolaridade, saneamento, emprego e renda, etc.) }\end{array}$ & & & \\
\hline
\end{tabular}

Para cada categoria avaliada pode-se defini-lo como existente, inexistente ou parcialmente existente. Nas colunas de Explicitação do conteúdo do EIA quando foi assinalado o número 1 significa que um dos EIA recebeu aquela classificação, número 2 quando em dois EIA recebeu aquela classificação, e 0 quando não foi identificada em nenhum EIA. 


\section{RESULTADOS E DISCUSSÃO}

A matriz apresentada na Tabela 1 condensa os resultados obtidos na análise, demonstrando a existência, inexistência ou a existência parcial das categorias avaliadas nos EIA analisados.

Tabela 1 - Resultados da Matriz de análise das questões de saúde nos estudos de impacto ambiental da Samarco para a barragem do Fundão (2005) e para o Sistema de Disposição de Rejeito - Alegria Sul (2016)

\begin{tabular}{|c|c|c|c|}
\hline \multirow[t]{2}{*}{ Categoria Operacional } & \multicolumn{3}{|c|}{ Explicitação do conteúdo no EIA } \\
\hline & Inexistente & $\begin{array}{c}\text { Parcialmente } \\
\text { Existente }\end{array}$ & Existente \\
\hline \multicolumn{4}{|c|}{ DESCRIÇÃO DO PROJETO } \\
\hline $\begin{array}{l}\text { Participação de profissionais com perfil específico da } \\
\text { área de saúde }\end{array}$ & 2 & & \\
\hline $\begin{array}{l}\text { Inventário das substâncias químicas relacionadas ao } \\
\text { empreendimento e os riscos à saúde associados }\end{array}$ & & 2 & \\
\hline $\begin{array}{l}\text { Estimativas de aplicação de recursos financeiros, } \\
\text { direta ou indiretamente, na melhoria da saúde }\end{array}$ & 1 & & 1 \\
\hline \multicolumn{4}{|c|}{ DIAGNÓSTICO DO MEIO SOCIOECONÔMICO } \\
\hline $\begin{array}{l}\text { Características gerais da população } \\
\text { densidade, distribuição, idade e sexo, e tendências } \\
\text { demográficas) }\end{array}$ & & 1 & 1 \\
\hline $\begin{array}{l}\text { 'Dinâmica de ocupação e uso do território, migrações } \\
\text { e deslocamentos populacionais }\end{array}$ & & 1 & 1 \\
\hline $\begin{array}{l}\text { Condições de habitação, situação de emprego, } \\
\text { infraestrutura de transportes, assistência social e } \\
\text { condições e acesso a serviços públicos }\end{array}$ & & 1 & 1 \\
\hline $\begin{array}{l}\text { Acesso a serviços e estabelecimentos de saúde e a } \\
\text { capacidade de suporte (infraestrutura, número de } \\
\text { leitos, profissionais de saúde, etc.) }\end{array}$ & & 1 & 1 \\
\hline $\begin{array}{l}\text { Perfil epidemiológico da população (difusão e } \\
\text { propagação de doenças, sua frequência, seu modo de } \\
\text { distribuição, sua evolução, etc.) }\end{array}$ & 2 & & \\
\hline $\begin{array}{l}\text { Indicadores comportamentais, incluindo a dieta, o } \\
\text { tabagismo, a atividade física e o uso de álcool }\end{array}$ & 2 & & \\
\hline $\begin{array}{l}\text { Condições ambientais como a qualidade do ar, água e } \\
\text { solo, a presença de áreas contaminadas }\end{array}$ & & & 2 \\
\hline $\begin{array}{l}\text { Geração de insegurança - Percepção da população } \\
\text { sobre os efeitos na saúde decorrentes dos impactos } \\
\text { ambientais do empreendimento }\end{array}$ & 2 & & \\
\hline $\begin{array}{l}\text { Geração de expectativa - Percepção da população } \\
\text { sobre as medidas de segurança e garantias de } \\
\text { investimentos na saúde }\end{array}$ & 1 & 1 & \\
\hline \multicolumn{4}{|c|}{ AVALIAÇÃO DE IMPACTOS AMBIENTAIS } \\
\hline $\begin{array}{l}\text { Identificação dos impactos à saúde dos trabalhadores } \\
\text { e da população residente no entorno do } \\
\text { empreendimento }\end{array}$ & 2 & & \\
\hline $\begin{array}{l}\text { Impactos sobre indicadores de saúde (morbidade, } \\
\text { mortalidade, atendimentos ambulatorial e hospitalar, } \\
\text { etc.) }\end{array}$ & 2 & & \\
\hline $\begin{array}{l}\text { Alteração na qualidade do solo, na qualidade da água } \\
\text { e do ar }\end{array}$ & & & 2 \\
\hline $\begin{array}{l}\text { Impactos sobre indicadores sociais e econômicos } \\
\text { (PIB, escolaridade, saneamento, emprego e renda, } \\
\text { etc.) }\end{array}$ & & 2 & \\
\hline
\end{tabular}


No que diz respeito à Descrição do Projeto, não foi identificada a participação de profissionais de saúde constantes do elenco oficial desses profissionais, com exceção dos biólogos na elaboração de nenhum dos EIA. De acordo com o Ministério da Saúde, são considerados profissionais de saúde de nível superior os graduados nas seguintes profissões: assistentes sociais, biólogos, biomédicos, profissionais de educação física, enfermeiros, farmacêuticos, fisioterapeutas; fonoaudiólogos, médicos, médicos veterinários, nutricionistas, odontólogos, psicólogos e terapeutas ocupacionais (BRASIL,1998) Havia somente uma equipe multidisciplinar habilitada, com registro profissional regulamentado por lei, características essas previstas na legislação (BRASIL,1986), mas com predomínio de profissionais de biologia, engenharia e geologia.

A apresentação das emissões, efluentes líquidos e resíduos gerados foi breve sem tampouco relaciona-los aos possíveis danos e agravos à saúde. Também não foram caracterizadas detalhadamente as substâncias químicas envolvidas e nem informadas suas periculosidades, classes, subclasses e quantidades totais. De acordo com Cancio (2008), o inventário das substâncias químicas é importante no sentido de identificar os possíveis riscos delas advindos.

Somente o EIA referente à Alegria Sul apresentou as estimativas de aplicação de recursos financeiros na melhoria da saúde, ao demonstrar as despesas referentes à saúde nos municípios da área de influência do empreendimento.

O diagnóstico do meio socioeconômico é fundamental permitir o conhecimento da realidade dos municípios que integram a área de influência do empreendimento. A partir dele pode-se chegar a um prognóstico sobre os possíveis impactos decorrentes da implantação e operação de uma atividade em relação às realidades sociais e econômicas de um dado território. Ambos os estudos traziam dados geográficos, demográficos, sociais, econômicos e de infraestrutura dos municípios integrantes das áreas de influência das atividades, com graus diferentes de detalhamento. Os diagnósticos utilizaram dados secundários oriundos de instituições de ensino e pesquisa, como o Instituto Brasileiro de Geografia e Estatística (IBGE). Complementarmente foram incluídos dados primários resultantes de pesquisas de percepção dos principais atores sociais residentes nas áreas de atuação dos empreendimentos, ambas realizadas pela Samarco.

No que diz respeito às características gerais da população, dinâmica de ocupação e uso do solo, condições de habitação, empregabilidade, estrutura de transporte, assistência social, acesso e condições dos serviços públicos e acesso à rede de serviços de saúde houve apresentação parcial no EIA da barragem de Fundão, com presença de análise quantitativa envolvendo dados estatísticos, em números absolutos e relativos, com detalhamento e discussão técnica superficial. Já no EIA para o sistema de Alegria-Sul, tais informações foram apresentadas também de maneira insuficiente mas acompanhadas por análises mais detalhadas que no EIA de Fundão.

No EIA de Fundão identificou-se somente a indicação de dados secundários quantitativos em tabelas referentes aos estabelecimentos de saúde (total, públicos e privados) existentes em Mariana e Ouro Preto, Minas Gerais, municípios da área de influência do empreendimento. Não há nenhum tipo de argumentação, comparação ou análise pormenorizada. Não foi discutida a questão dos fatores determinantes e condicionantes de saúde, tendo sido apresentados somente dados estatísticos secundários em uma tabela contendo o quantitativo de domicílios atendidos por esgotamento sanitário, abastecimento de água e destino dos resíduos sólidos coletados.

Já o EIA de Alegria-Sul trouxe informações mais detalhadas sobre a infraestrutura dos serviços de saúde dos municípios de Mariana e Ouro Preto, sendo apresentadas informações quantitativas sobre os estabelecimentos prestadores de serviços nos referidos municípios, a oferta de profissionais de saúde e leitos hospitalares e a disponibilidade de meios de transporte de pacientes. A maior parte das informações quantitativas estavam acompanhadas de análises contextualizadas que, ainda que superficiais e incompletas, aproximavam os dados apresentados da realidade vivida nos municípios da área de influência do empreendimento. Além disso, o estudo discutiu questões relacionadas ao repasse de verbas para o setor saúde, as condições de saneamento locais e, de maneira sucinta, a análise da percepção de gestores públicos em relação à qualidade dos serviços prestados. O EIA explicitava, também, uma análise de dados sobre as principais causas de morbimortalidade da população de modo geral e da mortalidade infantil na área de influência.

Conforme colocado por Valencio (2019) em cenários de crise surge uma onda inesperada de atendimentos prioritários de saúde devido aos agravos que ocorrem imediatamente após a ocorrência dos desastres (atendimento de vítimas de afogamentos, escoriações, cortes, choques, etc.), todos 
considerados atendimentos adicionais que não estavam previstos na rotina de atendimentos das unidades de saúde. Além disso há uma sobrecarga que ocorre em função dos danos às infraestruturas físicas de atendimento. Pacientes que eram atendidos em unidades de saúde dos territórios atingidos pela lama são direcionados para outras localidades devido à inviabilização do atendimento na área destruída.

Nenhum dos estudos contemplou adequadamente informações do perfil epidemiológico da população e tampouco análises e discussões técnicas sobre indicadores comportamentais. Cabe destacar que informações secundárias sobre o perfil epidemiológico da população estão disponíveis em dados secundários de livre acesso nos sistemas do Ministério da Saúde, como o Sistema de Informação de Agravos de Notificação (SINAN) e no Departamento de Informática do SUS (Datasus).

Os dois EIA analisados contemplaram informações acerca das condições ambientais envolvendo qualidade do ar, água e solo, bem como a presença de áreas contaminadas. Apesar disso, não apresentaram análises abrangentes em relação aos fatores ambientais e sua possível interferência na saúde humana. É muito importante uma análise ambiental abrangente desses quesitos, em consonância com o preconizado pela Vigilância em Saúde Ambiental (VSA) que busca compreender a relação da questão ambiental com a saúde, por intermédio da vigilância dos fatores de risco ambientais que possam interferir na saúde (BRASIL, 2017).

As análises de percepção da população sobre geração de insegurança e de expectativa foram obtidas por intermédio de entrevistas com atores sociais locais realizadas pela própria Samarco na área de influência dos empreendimentos. Como esses estudos foram desenvolvidos em momentos distintos, um antes do desastre e outro após o desastre, foram identificadas variações nessas percepções. Após o desastre ficou clara a sensação de anseio e insegurança da população residente na área de influência da barragem, tendo havido relatos envolvendo mudanças no estilo de vida, perda da qualidade da água, perda de laços comunitários, mortandade de animais e proliferação de insetos (ARCADIS, 2016). Apesar de ter havido coleta de dados acerca da geração de insegurança nos dois EIA, em nenhum deles foi contemplada uma análise específica de percepção relacionando as atividades da mineradora e seus possíveis impactos sobre a saúde humana. No que tange à percepção da população acerca das medidas de segurança, somente no EIA de Alegria Sul houve análise, sem no entanto, abordar informações sobre investimentos na área de saúde.

Os impactos à saúde da população não se limitam à situação imediata do rompimento da barragem, mas se desdobram em processos duradouros de crise social, frequentemente intensificada pelos encaminhamentos institucionais que Ihe são dirigidos, ao crescente cansaço provocado pelos processos burocráticos e pela sensação de insegurança em relação à retomada de suas vidas comunitárias (ZHOURI et al., 2018).

Nos capítulos referentes à Avaliação de Impactos Ambientais nenhum dos estudos previu adequadamente os impactos e riscos à saúde humana, seja dos trabalhadores, seja dos residentes no entorno do empreendimento. Pode-se observar avaliações superficiais com ausência parcial ou total de análises associadas. Tais informações, por apresentadas fora de um contexto social não foram capazes de demonstrar de forma sistemática as possíveis interferências do empreendimento na realidade vivida pela população, o alcance espacial dos danos socioambientais, e de que forma estes impactos poderiam causar danos à saúde humana. Apresentados dessa forma, o conteúdo sobre saúde dos estudos analisados difere dos pressupostos de Vigilância em Saúde Ambiental (VSA) conforme estabelecido pelo Ministério da Saúde (BRASIL, 2017).

Os capítulos de Avaliação de Impactos Ambientais de ambos os EIA também não apresentaram prognósticos sobre os impactos dos empreendimentos em relação aos indicadores de saúde. Considerando que trabalhadores da própria Samarco e, também, de empresas terceirizadas prestadoras de serviços foram vitimados no rompimento da barragem de Fundão, e que tratou-se de um evento agudo que causou lesões corporais, sequelas irreversíveis, efeitos crônicos, sofrimento psíquico e mortes, a adoção de mecanismos de investigação dos impactos à saúde e o amadurecimento das preocupações envolvendo as condições de trabalho, saúde e ambiente são fundamentais e, mesmo, esperados.

O rompimento de uma barragem de contenção de rejeitos de mineração pode levar a contaminação e sedimentação de cursos d'água, à contaminação do ar e do solo, à alteração dos ciclos de hospedeiros, reservatórios e vetores de enfermidades, a danos às habitações e à infraestrutura e a uma diversidade de impactos socioeconômicos. Tais fatos podem desencadear uma série de agravos 
e enfermidades, dentre os quais pode-se destacar transtornos de saúde mental, dermatites, parasitoses, zoonoses, arboviroses, problemas respiratórios, dentre outros (FREITAS et al., 2019).

O rompimento alterou completamente a rotina das comunidades, o funcionamento dos municípios e das regiões afetadas, criando uma sensação de medo e insegurança em função da situação de penúria à qual ficaram submetidas as populações atingidas. Diante desse cenário de sofrimento social, a saúde mental e o bem-estar psicossocial dos atingidos são pontos prioritários a serem acompanhados, sendo necessárias ações coordenadas entre os atores de órgãos governamentais e não governamentais envolvidos (SANTOS et al., 2019).

A avaliação de impacto dos empreendimentos na qualidade do ar, solo, água e indicadores socioeconômicos foi parcialmente contemplada nos dois EIA analisados, mas o ideal seria uma análise ampliada, principalmente com maior foco na epidemiologia.

Do ponto de vista metodológico da avaliação de impactos, os estudos analisados evidenciaram a insuficiência dos métodos adotados para realizar um prognóstico sobre os impactos à saúde da população e suas implicações e desdobramentos sobre os indicadores ambientais e socioeconômicos. Além disso, ainda que o EIA de Alegria-Sul tenha abordado o tema saúde de maneira mais detalhada quando comparado ao EIA de Fundão, não foi efetuada uma análise aprofundada sobre os efeitos potenciais das intervenções a serem realizadas pelo empreendimento sobre a saúde das pessoas residentes na área de influência. A falta de previsão dos possíveis impactos ambientais dificulta que sejam propostas medidas de prevenção, controle e mitigação desses impactos.

\section{CONSIDERAÇÕES FINAIS}

Em face do exposto pode-se perceber que os EIA não possibilitaram que fosse efetuado um prognóstico eficiente sobre os possíveis impactos à saúde da população bem como sobre as implicações sobre os indicadores socioeconômicos e ambientais da região.

A apresentação de dados e as discussões foram superficiais e insuficientes, desprezando a presença de categorias de profissionais de saúde, sem tomar como base a realidade epidemiológica local e sem considerar possíveis fatores determinantes e condicionantes de saúde.

O presente estudo permitiu observar o desafio que é a inserção da saúde no processo de licenciamento ambiental. A este desafio soma-se os retrocessos institucionais que o Brasil vem vivenciando, com destaque para o desmantelamento dos sistemas de regulação ambiental e a tentativa de desmobilização, criminalização e retirada de direitos dos grupos engajados na resistência à mineração. Isso inclui tentativas de mudanças na legislação vigente visando simplificar e tornar mais célere o processo de licenciamento ambiental. E isso tudo vem ocorrendo a despeito da multiplicação de desastres ambientais causados pelo rompimento de barragens (ZHOURI, 2018).

$\mathrm{Na}$ verdade, o que se observa, de maneira geral, é a grande fragilidade nos processos de licenciamento ambiental de empreendimentos potencialmente poluidores, com falta de estrutura técnica, financeira e política nos órgãos ambientais oficiais para a análise de riscos, para o questionamento dos relatórios produzidos pelas empresas e para exigir melhores soluções para a proteção ambiental, a saúde dos trabalhadores e das populações (LACAZ et al., 2017). Especificamente no caso da mineração, além dessas fragilidades do processo de licenciamento, devem ser considerados a manutenção do método obsoleto e perigoso de construção de barragens por alteamento a montante, a debilidade na fiscalização destas estruturas e a baixa capacidade de preparação e resposta ao rompimento de barragens pelos municípios vulneráveis a esse risco (FREITAS et al., 2019).

O rompimento da barragem em Mariana, MG, comprometeu o auto sustento e a renda familiar das pessoas, levou à perda de laços afetivos, gerou um clima de tensão e incertezas em relação à reparação dos danos, causou mortes e danos não letais à saúde, inclusive com comprometimento psicológico.

Com o rompimento da barragem, 40 municípios foram afetados em dois estados, Minas Gerais e Espírito Santo, totalizando 663 quilômetros de corpos hídricos diretamente impactados (VORMITTAG et al, 2018). Portanto, o rompimento traz em si uma dimensão complexa que mobiliza quase toda a estrutura do setor de Saúde Pública (FREITAS, 2018), o que requer a implementação de estratégias 
de monitoramento e acompanhamento de longo prazo em todos os níveis de atenção à saúde (PINHEIRO; SILVA, 2019).

As discussões sobre a possibilidade de uma avaliação multidisciplinar, que considere os impactos da modificação do meio ambiente sobre a saúde da população, especialmente, durante a implantação de projetos de desenvolvimento potencialmente poluidores, ainda é incipiente (SILVEIRA; ARAÚJO, 2014). Assim, urge a necessidade de estabelecimento de um novo marco regulatório que leve ao aumento e fortalecimento da participação da temática saúde no processo de licenciamento ambiental.

Muito foi discutido sobre os temas saúde e ambiente após a ocorrência dos desastres nas cidades de Mariana e Brumadinho. Um futuro seguro e sustentável exige atenção e aprendizagem, entretanto, não se reduz a atitudes puramente reflexivas sobre as "lições aprendidas para a catástrofe não se repetir". O histórico dos desastres industriais mostra que a capitalização dos ensinamentos de acidentes não ocorre, pelo menos, completamente, e que em geral, estas lições são esquecidas (LLORY; MONTMAIEUIL, 2014). É imprescindível a busca ativa por mudanças por parte das empresas e dos órgãos governamentais. Não se deve limitar apenas a relatar os desastres e suas repercussões, são necessárias propostas concretas de modificação do atual modelo de desenvolvimento, licenciamento ambiental e fiscalização das barragens de mineração para que desastres como estes não voltem a ocorrer.

O estudo comparativo entre os dois EIA permitiu concluir que a inserção das questões de saúde nos dois estudos ofereceu resultados sobre a inserção da saúde similares e incipientes, com leve superioridade do EIA de Alegria Sul. A pesquisa ajudou a compreender as fragilidades que existem na participação do setor de saúde no licenciamento ambiental de barragens de rejeitos de mineração.

\section{REFERÊNCIAS}

ARCADIS. Estudo de Impacto Ambiental (EIA). Sistema de Disposição de Rejeitos - Alegria Sul. Ouro Preto: ARCADIS, 2016.

BARBOSA, E. M.; BARATA, M. M. L.; HACON, S. S. A saúde no licenciamento ambiental: uma proposta metodológica para a avaliação dos impactos da indústria de petróleo e gás. Ciência e Saúde Coletiva, v. 17,n. 2, p. 299-310, 2012. https://doi.org/10.1590/S1413-81232012000200005

BARBOSA, F. A. R. et al. O desastre de Mariana e suas consequências sociais, econômicas, políticas e ambientais: porque evoluir da abordagem de Gestão dos recursos naturais para Governança dos recursos naturais? In: Arquivos do Museu de História Natural e Jardim Botânico, v.1. Belo Horizonte: UFMG, Museu de História Natural, 2010.

BARDIN, L. Análise de conteúdo. Lisboa: Edições 70, 1977.

BORGES, S.; O desastre da barragem de rejeitos em Mariana, Minas Gerais: aspectos socioambientais e de gestão na exploração de recursos minerais. Cuadernos de Geografia: Revista Colombiana de Geografia, v. 27, n. 2, p. 301-312, 2018. https://doi.org/10.15446/rcdg.v27n2.63008

BRASIL. Lei $n^{\circ}$ 6.938, de 31 de agosto de 1981. Dispõe sobre a Política Nacional de Meio Ambiente, seus fins e mecanismos de formulação e aplicação e dá outras providências. Disponível em: http://www.planalto.gov.br/ccivil 03/Leis/L6938.htm. Acesso em: 07 jul. 2019.

BRASIL. Conselho Nacional de Saúde. Resolução no 01, de 23 de janeiro de 1986. Dispõe sobre critérios básicos e diretrizes gerais para o Relatório de Impacto Ambiental - RIMA. Diário Oficial [da] República Federativa do Brasil, Brasília, DF, 17 fev. 1986.

BRASIL. Ministério da Saúde. 2017. Vigilância Ambiental. Disponível em: < http://www.saude.gov.br/vigilancia-em-saude/vigilancia-ambiental>. Acesso em: 13 mar. 2019.

BRASIL. Conselho Nacional de Saúde. Resolução $n^{\circ}$ 287, de 08 de outubro de 1998. Disponível em: http://conselho.saude.gov.br/docs/Reso287.doc. Acesso em: 06 jul. 2019.

CANCIO, J.A. Inserção das questões de saúde no estudo do impacto ambiental. Dissertação [Mestrado]. Universidade Católica de Brasília. Brasília, 2008.

FIGUEIREDO, N.M.A. Método e metodologia na pesquisa científica. 2 ed. São Caetano do Sul, SP: Yendis Editora, 2007. 
FREITAS, C. M. de et al. Da Samarco em Mariana à Vale em Brumadinho: desastres em barragens de mineração e Saúde Coletiva. Cadernos de Saúde Pública, v. 35, n. 5, e00052519, 2019. https://doi.org/10.1590/0102-311x00052519

FREITAS, C. M. Guia de preparação e respostas do setor saúde aos desastres. Rio de Janeiro, RJ: FIOCRUZ/Secretaria de Vigilância em Saúde, 2018.

FREITAS, C. M.; AMORIM, A. E. Vigilância ambiental em saúde de acidentes químicos ampliados no transporte rodoviário de cargas perigosas. Informe Epidemiológico do Sus, v. 10, n. 1, p. 31-42, 2001. https://doi.org/10.5123/S0104-16732001000100004

GMG. Gabinete Militar do Governador. Coordenadoria Estadual de Defesa Civil. Informações: Desastre Barragem de rejeitos de Brumadinho - 03/04/2019. [online] Minas Gerais. 2019 Abril 03. Disponível em: http://www.defesacivil.mg.gov.br/index.php/component/gmg/page/634INFORMACOES0304>. Acesso em: 12 abr. 2019.

IBAMA. Instituto Brasileiro do Meio Ambiente e dos Recursos Naturais Renováveis. Rompimento da Barragem de Fundão: Documentos relacionados ao desastre da Samarco em Mariana / MG. [online] 2016 Mar 16. Disponível em: <https://www.ibama.gov.br/recuperacao-ambiental/rompimentoda-barragem-de-fundao-desastre-da-samarco/documentos-relacionados-ao-desastre-da-samarco-emmariana-mg >. Acesso em: 12 abr. 2019.

LACAZ, F. A. C.; PORTO, M. F. S.; PINHEIRO, T. M. M. Tragédias brasileiras contemporâneas: o caso do rompimento da barragem de rejeitos de Fundão / Samarco. Revista Brasileira de Saúde Ocupacional, v.42, n.9, p.1-12, 2017. https://doi.org/10.1590/2317-6369000016016

LLORY M.; MONTMAIEUIL, M. O acidente e a organização. Belo Horizonte: Fabrefactum, 2014.

LOZANO, F. A. E. Seleção de locais para barragens de rejeitos usando o método de análise hierárquica. 2006. 128 f. Dissertação (Mestrado em Engenharia Geotécnica), Departamento de Engenharia de Estruturas e Fundações, USP, São Paulo, SP

MANSUR, S.M. et al. Antes fosse mais leve a carga: introdução aos argumentos e recomendações referente ao desastre da Samarco/ Vale/ BHP Hilton. IN: MILANEZ et al. A questão mineral no Brasil - Vol. 2. Antes fosse mais leve a carga: Reflexões sobre o desastre da Samarco/ Vale/ BHP Hilton. / Marcio Zonta e Charles Trocate (Orgs.) - Marabá, PA: Editorial iGuana., 2016. p. 17-50.

MILANEZ, B.; SANTOS, R.; MANSUR, S.M. A firma e suas estratégias coorporativas no pós-boom das commodities. In :MILANEZ et al. A questão mineral no Brasil - Vol. 2. Antes fosse mais leve a carga: Reflexões sobre o desastre da Samarco/ Vale/ BHP Hilton. / Marcio Zonta e Charles Trocate (Orgs.) - Marabá, PA: Editorial iGuana. 2016. p. 51-86.

MINAYO, M. C. S. (Org.). Pesquisa social: teoria, método e criatividade. Petrópolis: Vozes, 2001.

MINISTÉRIO DA SAÚDE. Secretaria de Vigilância em Saúde. Avaliação de Impacto à Saúde - AIS: metodologia adaptada para aplicação no Brasil. Brasília: Ministério da Saúde, 2014.

PORTO, M. F.S. A tragédia da mineração e do desenvolvimento no Brasil: desafios para a saúde coletiva. Cadernos de Saúde Pública, Rio de Janeiro, v. 32, n.2, e00211015, 2016. https://doi.org/10.1590/0102-311X00211015

RICO, M.; BENITO, G.; SALGUEIRO, A.R.; D'IEZ-HERRERO A.; PEREIRA, H.G. Reported tailings dam failures. A review of the european incidents in the worldwide context. Journal of Hazardous Materials, v.152, p.846-852, 2008. https://doi.org/10.1016/j.jhazmat.2007.07.050

SANTOS, M. A. L. et al. O cuidado em saúde mental da população de atingidos na tragédia da Samarco: reflexões a partir da práxis. In: Mar de lama da Samarco na bacia do rio Doce: em busca de respostas. PINHEIRO, T. M. M. et al (Org.). Belo Horizonte: Instituto Guaicuy; 2019. p. 190-198.

SIAM. Sistema Integral de Informação Ambiental. Acesso de visitantes. 2019. Disponível em: <http://www.siam.mg.gov.br/siam/processo/index.jsp> Acesso em: 10 fev. 2018.

SILVEIRA, M.; ARAÚJO NETO, M. D. Licenciamento ambiental de grandes empreendimentos: conexão possível entre saúde e meio ambiente. Ciência \& Saúde Coletiva, v. 19, n. 9, p. 3829-3838, 2014. https://doi.org/10.1590/1413-81232014199.20062013 
SORDIA, D. S.; DIAZ, Vicente I. Criterios de salud en la evaluación del impacto ambiental de proyectos de desarrollo. Revista Cubana Higiene Epidemiologia, v. 37, n.1, p.25-31, 1999.

VALENCIO, N. Nexos entre desastres ditos "naturais" e a Atenção Básica do SUS: a multidirecionalidade da crise. IN: RIBEIRO, Eduardo Augusto Werneck (Org.). Novos temas para se pensar as pesquisas em Geografia da Saúde. 1. ed. Blumenau: Instituto Federal Catarinense, 2019. p. 8-17.

VORMITTAG, E. M. P. A. A.; OLIVEIRA, M. A. DE; GLERIANO, J. S. Health evaluation of the Barra Longa population affected by the disaster in Mariana county. Ambiente e Sociedade., v. 21, e01222, 2018. https://doi.org/10.1590/1809-4422asoc0122r2vu1811ao

ZHOURI, A. Produção de conhecimento num 'campo minado. In: ZHOURI, A. (Org.). Mineração: violências e resistências: um campo aberto à produção de conhecimento no Brasil. 1.ed. Marabá, PA: Editorial iGuana; ABA, 2018. p. 8-26. 